ORIGINAL ARTICLE

\title{
Angiogenic effect of intramuscular administration of basic and acidic fibroblast growth factor on skeletal muscles and influence of exercise on muscle angiogenesis
}

\author{
A Efthimiadou, B Asimakopoulos, N Nikolettos, A Giatromanolaki, E Sivridis, D N Papachristou, \\ E Kontoleon
}

Br J Sports Med 2006;40:35-39. doi: 10.1136/bjsm.2005.018754

See end of article for authors' affiliations

.....................

Correspondence to: Dr Efthimiadou,

Department of Physiology, Democritus University of Thrace, Medical School, University Campus, Dragana, Alexandroupolis 68100, Greece; aefthim@ med.duth.gr

Accepted 6 June 2005
Background: Angiogenic factors which control the angiogenic process represent a promising strategy for restoration of blood flow, but require further evaluation before clinical use. Exercise has also been reported to induce neovascularisation in muscles.

Objectives: To evaluate the angiogenic effects of basic fibroblast growth factor (b-FGF) and acidic fibroblast growth factor (a-FGF) on rat gastrocnemius muscle, when administered intramuscularly, and to compare them with those obtained by daily exercise.

Methods: Forty nine rats were allotted to the following groups: $A$, controls; $B$, exercise by swimming; $C 1$ and $C 2$, intramuscular injection of b-FGF and a-FGF respectively; D1 and D2, b-FGF and a-FGF injection in combination with exercise. The antibody mouse anti-rat CD31 was used to evaluate the numbers of blood vessels present in histological preparations of gastrocnemius muscle.

Results: Significant increases in the numbers of blood vessels of the right gastrocnemius muscles in groups $C 1$ and D1 were observed compared with controls $(p<0.05)$. There was only a slight increase in angiogenesis in the left gastrocnemius muscle of groups $C 1$ and Dl compared with controls ( $>>0.05$ ), and there was a decrease in angiogenesis in the gastrocnemius muscle of the swimming group compared with controls.

Conclusion: The intramuscular administration of b-FGF, but not a-FGF, induced significant local angiogenesis in gastrocnemius muscle at the site of injection.
A ngiogenesis is a complex process involving sprouting of endothelial cells from pre-existing microvessels and their subsequent development into new vessels. It normally occurs during embryonic growth, but also in certain conditions in adult life. ${ }^{1}$ Formation of new vessels in the endometrium of women during their monthly menstrual cycle is one such example. Furthermore, exercise can lead to changes in muscle mass, a process associated with angiogenesis. $^{2}$

In addition to the physiological events related to angiogenesis, a variety of pathological conditions are known to be associated with it, including diabetic retinopathy, malignant neoplasms, rheumatoid arthritis, and angiomas. ${ }^{3}$

Fibroblast growth factors (FGFs) and the FGF receptor system available today for treatment of clinical conditions associated with ischaemia represent a promising strategy for restoration of blood flow and require further evaluation before extensive clinical use. ${ }^{4}$ The FGFs are a family of at least 20 peptides that have angiogenic properties. Initially, FGFs were isolated from the brain by Gospodarowicz in 1974. ${ }^{5}$ Since then, various peptides of similar structure in various tissues have been found to manifest similar angiogenic properties. ${ }^{4}$ Acidic (a)-FGF is a 140 amino acid polypeptide which has 55\% homology with basic (b)-FGF, which consists of 154 amino acid residues. Both peptides are expressed in a variety of tissues including brain, pituitary, myocardium, kidney, and liver as well as macrophages and endothelial and muscle cells. FGFs act as protein mitogens stimulating angiogenesis under various physiological and pathological conditions. Physiologically, they are also implicated in brain development, cartilage formation, soft tissue repair, migration, and differentiation of cells of mesenchymal or neuroectodermal origin. These factors are also implicated in pathological processes, such as neoplasms, as they induce the neoangiogenesis associated with tumour growth. ${ }^{67}$ Although angiogenic therapy using recombinant growth factors holds much hope for the treatment of ischaemic diseases, there are still many unanswered questions, including the best method of administration, the correct dose, and the duration of the treatment.

As the use of recombinant angiogenic growth factors holds much hope for the treatment of ischaemic diseases, the aims of this study were to evaluate if the intramuscular injection of b-FGF and a-FGF into the right gastrocnemius muscle of the rat would induce local angiogenic effects in that muscle and to compare them with those achieved by exercise. The choice of the right gastrocnemius muscle was random.

\section{MATERIALS AND METHODS \\ Design}

Forty nine male Wistar rats (330-400 g body weight; age 4 months) were used for this study. During the experimental period, the animals lived under stable conditions of temperature and a reverse light cycle programme, and were allowed to eat ad libitum. They were divided into six groups (table 1).

Group A, consisting of 14 rats, were controls. This group was subdivided into: Al, seven rats to be compared with the swimming group; A2, seven rats in which $0.1 \mathrm{ml}$ saline was injected via a syringe of insulin under ether anaesthesia into

Abbreviations: a-FGF, acidic fibroblast growth factor; $b-F G F$, basic fibroblast growth factor; VEGF, vascular endothelial growth factor 


\begin{tabular}{ll}
$\begin{array}{l}\text { Table } 1 \\
\text { treatments }\end{array}$ & Experimental groups of rats and their \\
\hline Group & Treatment \\
\hline A1 & No treatment (controls) \\
A2 & Intramuscular saline (controls) \\
B & Swimming \\
C1 & Intramuscular b-FGF \\
C2 & Intramuscular a-FGF \\
D1 & Intramuscular b-FGF + swimming \\
D2 & Intramuscular a-FGF + swimming \\
\hline a-FGF, acidic fibroblast growth factor; b-FGF, basic \\
fibroblast growth factor.
\end{tabular}

the middle of the right gastrocnemius muscle every three days for 15 days.

Group B consisted of seven rats that swam in water of constant temperature $\left(36^{\circ} \mathrm{C}\right)$ every day for 15 days. The duration of the swim was five minutes on the first day and this increased progressively up to 60 minutes on day 10, after which it remained constant until the end of the experiment. ${ }^{8}$

Group $\mathrm{Cl}$ consisted of seven rats that received $1 \mu \mathrm{g}$ b-FGF injected via a syringe of insulin into the middle of the right gastrocnemius muscle under ether anaesthesia every three days, for 15 days. Group C2 consisted of seven rats that received an equimolar dose of a-FGF $(0.88 \mu \mathrm{g})$ instead of bFGF according to the same protocol.

Group Dl consisted of seven rats that received b-FGF as described for group $\mathrm{Cl}$ but in combination with swimming as described for group B. Group D2 consisted of seven rats that received a-FGF as in group $\mathrm{C} 2$ but in combination with swimming as in group B.

The rats were killed on the 18th day, and the gastrocnemius muscles from both legs were removed, weighed, and sent for histological examination.

\section{Histological examination}

The tissues were fixed in $10 \%$ formalin, embedded in paraffin, cut transversely into $3 \mu \mathrm{m}$ thick sections in the middle of the specimen, and studied by immunohistochemical techniques. ${ }^{9}$ To evaluate angiogenesis, blood vessels were detected by the use of a monoclonal mouse anti-rat antibody (CD31, clone: PECAM-1; Dako, Glostrup, Denmark), which recognises the surface antigen CD31 of endothelial cells of mice. The immunohistochemical method of alkaline antialkaline phosphate (APAAP) was used according to the manufacturer's instructions (Innovex Biosciences, Richmond, CA, USA). ${ }^{10}$ In brief, the sections were deparaffinised, rehydrated, and subjected to proteolysis with the protease enzyme, type XXIV, at $37^{\circ} \mathrm{C}$ for 20 minutes. The primary antibody was added to the sections under review at 1:10 dilution, for 30 minutes at room temperature. The sections were then washed in Tris buffered saline. Serum originating from the reaction of rabbit serum against mouse serum was used as a secondary antibody at a dilution of 1:50 for 30 minutes. After the sections had been rinsed with Tris buffered saline, the two last stages were repeated for 10 minutes each. Staining was achieved by incubation in new fuchsin solution for 20 minutes. Normal mouse IgG instead of primary antibody was used as a negative control.

After inspection of the gastrocnemius tissue sections at low power $(\times 40$ and $\times 1000)$, angiogenic activity was quantified by counting all vessels with clear lumen or linear shape per optical field, at $\times 200$ magnification. Single endothelial cells were not included. Counting was performed in all available optical fields of the tissue section, and then the three fields of highest vessel density (hot spots) were used to obtain a mean value, which was the final score for each case. The two pathologists, who performed the assessment on a conference microscope, were blinded to the group of animals. ${ }^{10}$

\section{Statistical analysis}

The following variables were analysed: body weight at the beginning and end of the experiment; weight of each gastrocnemius muscle (left and right leg); number of blood vessels per optical field in each gastrocnemius muscle. Descriptive statistics were calculated for all groups. Comparisons among groups were performed with the Mann-Whitney $U$ test and Kruskal-Wallis test using the Statistica 4.5 statistical package for Windows (StatSoft Inc, Tulsa, Oklahoma, USA). $\mathrm{p}<0.05$ was considered significant.

\section{RESULTS}

Effects of exercise, b-FGF, and a-FGF on angiogenesis in gastrocnemius muscles

There were no deaths from the repeated anaesthesia. Also no difference in angiogenesis was observed in the gastrocnemius muscles between the control groups Al and A2 (table 2, fig lA).

Comparison between groups $\mathrm{Al}$ and $\mathrm{B}$ revealed a significant reduction in the number of vessels in both right $(\mathrm{p}<0.05)$ and left $(\mathrm{p}<0.05)$ gastrocnemius muscles triggered by exercise (table 2, fig 1B). On the other hand, comparing the muscle weights between group Al and group B (table 3 ), a significant increase in the weight of both the left $(p<0.01)$ and right $(\mathrm{p}<0.01)$ muscle was triggered by exercise.

In group $\mathrm{Cl}$, the mean number of blood vessels was different in the right gastrocnemius muscle, where b-FGF was injected intramuscularly, from the left gastrocnemius muscle (table 2). Comparison of group $\mathrm{Cl}$ with controls (group A2) with regard to the vessels in the right and left gastrocnemius muscle reveals that b-FGF increased vascularisation significantly $(\mathrm{p}<0.05)$ and selectively only in the right muscle (table 2, fig $\mathrm{lC}$ ). Furthermore, in group $\mathrm{Cl}$, the number of vessels in the right and left muscles was significantly higher than in the muscles of group $B$ $(\mathrm{p}<0.005$ and $\mathrm{p}<0.05$ respectively $)$.

It was also observed that the number of vessels in the right muscle in group C2 was significantly higher than in group B $(\mathrm{p}<0.05)$ but not higher than in group A2 (table 2, fig $1 D)$. However, groups $\mathrm{C} 2$ and $\mathrm{B}$ did not show any differences in angiogenesis in the left gastrocnemius muscle.

The combined administration of b-FGF and swimming (group D1) resulted in different effects on the right and left gastrocnemius muscles (table 2). Compared with group A2, in group Dl vascularisation was significantly increased in the right muscle $(p<0.05)$ but not in the left one (fig lE). Similarly, the vessels in the right and left muscles of group Dl were significantly increased compared with those of group B $(\mathrm{p}<0.005$ and $\mathrm{p}<0.05$ respectively $)$.

In group D2, it was also observed that the number of vessels in only the right muscle, and not the left one, was significantly higher than in group $B(p<0.05)$ (table 2 , fig $1 F)$.

Swimming appears to significantly decrease the vascularisation of both right and left gastrocnemius muscles compared with controls, probably as a result of changes in muscle mass. The intramuscular injection of b-FGF significantly increased vascularisation locally in the right muscle into which it was injected, whereas the intramuscular injection of a-FGF did not result in vascularisation of the injected muscle. When there was simultaneous administration of angiogenic factors and swimming, only b-FGF caused a major local increase in vessels in the muscle injected. No angiogenic action of either b-FGF or a-FGF was observed on the left side perhaps because of the predominance of the effects of exercise. 

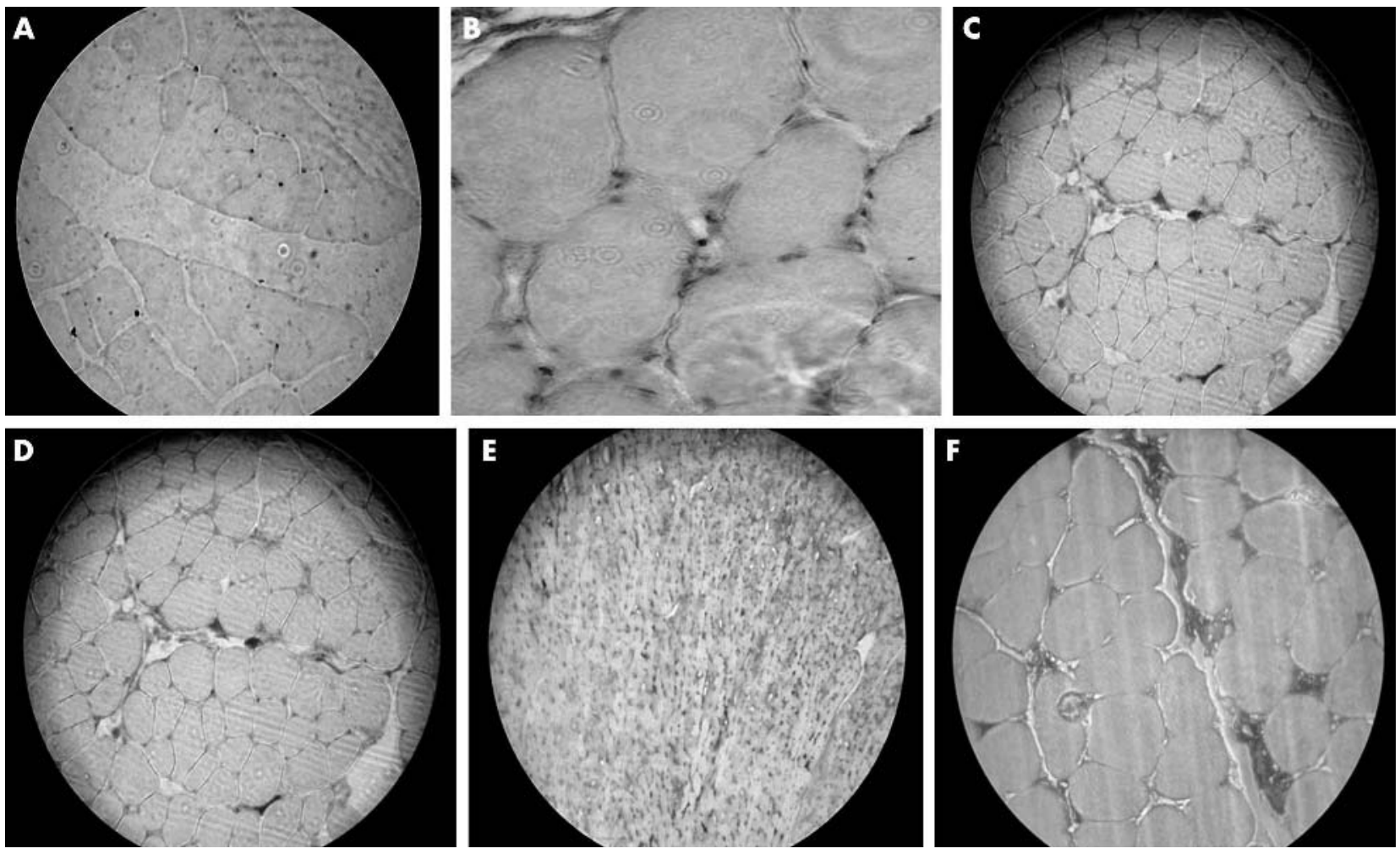

Figure 1 Immunohistochemical staining using monoclonal mouse anti-rat antibody (CD31, clone: INN-PECAM) of the blood vessels of rat gastrocnemius muscle. (A) Controls (groups A1 and A2). (B) Swimmers (group B). A significant decrease in muscle vasculature compared with the controls can be seen. (C) Rats that received intramuscular basic fibroblast growth factor (b-FGF) (group C1). A significant increase in muscle vasculature compared with the controls can be seen. (D) Rats that received intramuscular acidic fibroblast growth factor (a-FGF) (group C2). (E) Rats that received combined b-FGF and swimming (group D1). The maximum increase in blood vessels can be seen. (F) Rats that received combined a-FGF and swimming (group D2).

Effects of exercise, b-FGF, and a-FGF on the weight of rat gastrocnemius muscles

The weights of the right and left gastrocnemius muscles were also determined at the end of the experiment as an index of muscle mass (table 3). Exercise (group B) significantly increased the weight of the right $(\mathrm{p}<0.01)$ and left $(p<0.01)$ muscles compared with controls. The same significant increase in the weight of both the right

Table 2 Number of blood vessels per optical field in gastrocnemius muscle of the groups of rats studied

\begin{tabular}{lll}
\hline & \multicolumn{2}{l}{ Number of vessels per optical field } \\
\cline { 2 - 3 } Group & Left muscle & Right muscle \\
\hline A1 & $16.94(2.80)$ & $16.95(3.79)$ \\
A2 & $17.00(3.80)$ & $16.45(3.80)$ \\
$B$ & $12.70(3.00)^{*}$ & $12.82(2.80)^{*}$ \\
C1 & $18.40(3.30) \dagger$ & $20.80(2.40)^{*} \dagger$ \\
C2 & $15.70(2.98)$ & $17.20(3.10)^{\dagger}$ \\
D1 & $17.00(1.10) \dagger$ & $22.20(1.70)^{*} \dagger$ \\
D2 & $16.20(1.87)$ & $17.80(2.70) \dagger$ \\
\hline
\end{tabular}

Values are mean (SD)

* $p<0.05$ compared with controls.

$\mathrm{tp}<0.05$ compared with group $B$.

$A 1$, No treatment; $A 2,0.1 \mathrm{ml}$ saline injected into the right gastrocnemius muscle every three days for 15 days; $B$, made to swim every day for

15 days; $\mathrm{Cl}, 1 \mu \mathrm{g}$ basic fibroblast growth factor (b-FGF) injected into the right gastrocnemius muscle every three days, for 15 days; $\mathrm{C} 2$, an equimolar dose of acidic fibroblast growth factor (a-FGF; $0.88 \mu \mathrm{g}$ ) injected into the right gastrocnemius muscle every three days, for 15 days; D1, injected with b-FGF in combination with swimming; D2, injected with a-FGF in combination with swimming. $(\mathrm{p}<0.01)$ and left $(\mathrm{p}<0.01)$ muscles was observed in group $\mathrm{Cl}$ (intramuscular b-FGF) and group Dl (intramuscular bFGF plus swimming) compared with controls.

\section{DISCUSSION}

Angiogenesis, the creation of new vessels from pre-existing ones, is a complex process initiated under various physiological (embryonic growth, exercising muscles, menstrual cycle) and pathological (tumours, ischaemia, etc) conditions. ${ }^{6}$ Although the exact mechanisms underlying angiogenesis are

Table 3 Weight of the right and left gastrocnemius muscles in the groups of rats studied

\begin{tabular}{lll}
\hline Group & Right $(\mathbf{g})$ & Left $(\mathbf{g})$ \\
\hline A1 & $2.13(0.15)$ & $2.14(0.26)$ \\
A2 & $2.18(0.14)$ & $2.11(0.12)$ \\
B & $2.67(0.23)^{*}$ & $2.61(0.26)^{*}$ \\
C1 & $2.92(0.24)^{*}$ & $2.81(0.24)^{*}$ \\
C2 & $2.50(0.16)$ & $2.33(0.20)$ \\
D1 & $2.56(0.12)^{\star *}$ & $2.49(0.16)^{* *}$ \\
D2 & $2.49(0.18)$ & $2.45(0.15)$ \\
\hline
\end{tabular}

Values are mean (SD).

${ }^{*} \mathrm{p}<0.01$, ${ }^{* *} \mathrm{p}<0.05$ compared with controls.

$A 1$, No treatment; $A 2,0.1 \mathrm{ml}$ saline injected into the right gastrocnemius muscle every three days for 15 days; $B$, made to swim every day for

15 days; $\mathrm{Cl}, 1 \mu \mathrm{g}$ basic fibroblast growth factor (b-FGF) injected into the right gastrocnemius muscle every three days, for 15 days; $C 2$, an equimolar dose of acidic fibroblast growth factor (a-FGF; $0.88 \mu \mathrm{g}$ ) injected into the right gastrocnemius muscle every three days, for 15 days; D1, injected with b-FGF in combination with swimming; D2, injected with a-FGF in combination with swimming. 
What is already known on this topic

- It is known that receptors for fibroblast growth factors (FGFs) exist in organs such as brain, heart, and muscle

- Also, several studies have shown that FGFs have an angiogenic effect in pathological conditions, such as cancer but also under ischaemic conditions-for example, in the cardiac muscle

still unknown, over the last few years a variety of cytokines have been recognised to mediate this process. Some of these factors are now available through recombinant DNA technology and can be used in appropriate clinical cases. From the clinical point of view, angiogenesis today represents an interesting area of research in two different areas.

Firstly, tumour progression is characterised by excessive angiogenesis where vessels develop in an uncontrolled manner. The now generally accepted concept that growth of most types of tumours requires angiogenesis was put forward by Folkman and others. ${ }^{71}$ Secondly, ischaemia is a condition in which the blood supply to the cells is reduced, and angiogenesis may be a compensatory mechanism by which the problem can be bypassed. ${ }^{12}$ Strategies for increasing angiogenesis are applicable in this case. A large number of cytokines have been recognised to cause neovascularisation, but FGF and vascular endothelial growth factor (VEGF) have shown the best results in experimental models and clinical trials.

In one study, ${ }^{13}$ b-FGF was administered to dogs intramuscularly or intravenously, and the angiogenic effects on the limbs were observed independently of the method of administration. In the clinical study "TRAFFIC", ${ }^{14}$ b-FGF was infused intra-arterially into patients with intermittent claudication. Patients with moderate to severe intermittent claudication improved their exercise capacity, with no significant difference between those who received one dose and those who received several doses.

Current evidence suggests that angiogenesis is regulated through the balance between various stimulatory and inhibitory factors, and exercise can upregulate angiogenic factors, one of which may be b-FGF. ${ }^{15}$ In experiments on exercise training of skeletal muscles for example, VEGF has been shown to be a more important regulator of angiogenesis than b-FGF. ${ }^{16}$ Similar conclusions have been reported by others, who showed increased VEGF mRNA in skeletal muscles after exercise in rats $^{17}{ }^{18}$ and humans. ${ }^{19} 20$ Furthermore, experiments involving electrical stimulation or short term exercise training of skeletal muscles ${ }^{21} 22$ have suggested that VEGF and angiotensin II play an important role in exercise induced angiogenesis. Some data ${ }^{23}$ suggest that VEGF, except biological activities in endothelial cells, induce elongation and migration of desmin-positive pericytes and coverage of angiogenic capillaries. VEGF can also cause a significant decrease in intercapillary spaces, an indicator of intussusceptive vascular growth. Although most researchers agree that angiogenesis through exercise is mediated by angiogenic factors, other investigators ${ }^{24}$ have reported that exercise induced angiogenesis in skeletal muscles of rats with obstruction of the crural artery was not associated with changes in tissue content of b-FGF.

We have recently reported that both exercise and intramuscular administration of VEGF increases angiogenesis in rat heart, although only exercise alone increased angiogenesis very significantly. The combined protocol (administration of growth factor and exercise) led to an increase in angiogenesis in the cardiac muscles. ${ }^{25}$
What this study adds

- This study confirms that intramuscular administration of b-FGF increases locally blood vessels in skeletal muscle

- The administration of a-FGF is not effective

In this study, we injected b-FGF and a-FGF intramuscularly into the right gastrocnemius muscle. We observed a local angiogenic effect with b-FGF but the effect of a-FGF was negligible. Also exercise reduced the number of vessels per optical field in the skeletal muscles. As our method detects vessels per optical field, the only explanation of the above results is that increased muscle mass led to the artificial result of a reduction in angiogenesis. From the results of angiogenesis when FGF administration was combined with exercise, we can conclude that only b-FGF led to a local increase in angiogenesis in the injected muscle, with a-FGF only having slight local effect.

In conclusion, this study shows that intramuscular injection of b-FGF but not of a-FGF is effective in skeletal muscle only locally and at high concentrations. These observations are interesting with regard to the potential administration of b-FGF to alleviate conditions characterised by insufficient blood supply, such as limb ischaemia. Also, the study provides a basis for further application of b-FGF in humans for the treatment of ischaemic vascular lesions.

\section{Authors' affiliations}

A Efthimiadou, B Asimakopoulos, N Nikolettos, E Kontoleon, Department of Physiology, Democritus University of Thrace, Medical School, Alexandroupolis, Greece

A Giatromanolaki, E Sivridis, Department of Pathology, Democritus University of Thrace

D N Papachristou, Medicine-Division of Endocrinology, Democritus University of Thrace

Competing interests: none declared

\section{REFERENCES}

1 Favier J, Corvol P. Physiological angiogenesis. Therapie 2001;56:455-63.

2 Gavin TP, Robinson CB, Yeager RC, et al. Angiogenetic growth factor response to acute systemic exercise in human skeletal muscle. J Appl Physiol 2004;96:19-24.

3 Hamaham D, Folkman J. Patterns and emerging mechanisms of the angiogenetic switch during tumorigenesis. Cell 1996;86:353-64.

4 Auguste P, Javerzat S, Bikfalvi A. Regulation of vascular development by fibroblast growth factors. Cell Tissue Res 2003;314:157-66.

5 Gospodarowicz D. Localisation of FGF and its effect alone and with hydrocortisone on 3T3 cell growth. Nature 1974;249:123-7.

6 Carmellet $\mathbf{P}$. Mechanisms of angiogenesis and arteriogenesis. Nat Med 2000;6:389-95.

7 Folkman J, Handernschild C. Angiogenesis in vitro. Nature 1980;288:551-6.

8 Stergiou-Michailidou V, Kontoleon-Vakalopoulou E. The combined action of exercise and methylprednisolone sodium succinate on the rat gastrocnemius muscle. Int J Biochem 1991;23:231-4.

9 Shi SR, Key ME, Kalra K. Antigen retrieval in formalin-fixed, paraffinembedded tissues: an enhancement method for immunohistochemical staining based on microwave oven heating of tissue sections. J Histochem Cytochem 1991;39:741-8.

10 Giatromanolaki A, Koukourakis MI, Simopoulos C, et al. VEGF expression in operable gallbladder carcinomas. Eur J Surg Oncol 1991;29:879-83.

11 Cross MJ, Claesson-Welsh L. FGF and VEGF function in angiogenesis: signaling pathways, biological responses and therapeutic inhibition. Trends Pharmacol Sci 2001;22:201-7.

12 Amant C, Berthon L, Walsh K. Angiogenesis and gene therapy in man: dream or reality? Drugs 1999;59:33-6.

13 Ibukiyama Ch. Angiogenetic therapy using FGF and VEGF for ischemic vascular lesions. Jpn Heart J 1996;137:285-300.

14 Lederman RJ, Mendelsohn FO, Anderson RD, et al. TRAFFIC Investigators. Therapeutic angiogenesis with rFGF-2 for intermittent claudication (the TRAFFIC study): a randomized trial, Lancet 2002;359:2053-8. 
15 Gustafsson T, Kraus WE. Exercise-induced angiogenesis-related growth and transcript factors in skeletal muscle, and their modification in muscle pathology. Front Biosci 2001;1:D75-89.

16 Wagner PD. Skeletal muscle angiogenesis. A possible role for hypoxia. Adv Exp Med Biol 2001;502:21-38.

17 Breen EC, Johnos EC, Wagner $\mathrm{H}$, et al. Angiogenetic growth factor mRNA responses in muscle to a single bout of exercise. J Appl Physiol 1996;81:355-61.

18 Gavin TP, Spector DA, Wagner $\mathrm{H}$, et al. Nitric oxide synthase inhibition attenuates the skeletal muscle VEGF mRNA response to exercise. J Appl Physiol 2000;88:1192-8.

19 Richardson RS, Wagner $\mathrm{H}$, Mudaliar SR, et al. Exercise adaptation attenuates VEGF gene expression in human skeletal muscle. Am J Physiol Heart Circ Physiol 2000;279:772-8.

20 Gustafsson T, Puntschart A, Kaijser L, et al. Exercise-induced exression of angiogenesis-related transcription and growth factor in human skeletal muscle. Am J Physiol Heart Circ Physiol 1999;276:679-85.

21 Amaral SL, Papanek PE, Greene AS. Angiotensin 2 and VEGF are involved in angiogenesis induced by short-term exercise training. Am J Physiol Heart Circ Physiol 2001;281:1163-9.

22 Amaral SL, Linderman JR, Morse MM, et al. Angiogenesis induced by electrical stimulation is mediated by angiotensin 2. Microcirculation 2001;8:57-67.

23 Hagedorn M, Balke M, Schidt A, et al. VEGF coordinates interaction of pericytes and endothelial cells during vasculogenesis and experimental angiogenesis. Dev Dyn 2004;230:23-33.

24 Deschenes MR, Ogilvie RW. Exercise stimulates neovascularization in occluded muscle without affecting b-FGF content. Med Sci Sports Exerc 1999;31:1599-604.
25 Efthimiadou A, Asimakopoulos B, Nikolettos N, et al. The angiogenetic effect of intramuscular administration of VEGF on muscle. The influence of exercise on angiogenesis. In Vivo 2004;18:825-30.

\section{COMMENTARY}

This is a basically sound paper that concerns the investigation of whether intramuscular administration of b-FGF and aFGF can result in muscle angiogenesis, and whether any increase in angiogenesis caused by FGF administration is similar to the angiogenesis induced by exercise training. A statistically significant increase in angiogenesis resulted from intramuscular injection of b-FGF, but not from a-FGF injection or exercise alone. These observations may be of great importance in humans for the clinical treatment of ischaemic vascular lesions and for conditions characterised by insufficient blood supply.

T S Lialiaris

Department of Genetics, Democritus University of Thrace, Alexandroupolis 68100, Greece; lialiari@med.duth.gr

\section{bmjupdates+}

bmjupdates+ is a unique and free alerting service, designed to keep you up to date with the medical literature that is truly important to your practice.

bmjupdates+ will alert you to important new research and will provide you with the best new evidence concerning important advances in health care, tailored to your medical interests and time demands.

Where does the information come from?

bmjupdates+ applies an expert critical appraisal filter to over 100 top medical journals A panel of over 2000 physicians find the few 'must read' studies for each area of clinical interest

Sign up to receive your tailored email alerts, searching access and more...

www.bmjupdates.com 